\title{
Parallaxes of 16 Planetary Nebulae
}

\author{
Hugh C. Harris \\ U.S. Naval Observatory, 10391 W. Naval Obs. Rd., Flagstaff, AZ 86001, USA \\ email: hch@nofs.navy.mil
}

\begin{abstract}
Improved trigonometric parallaxes of 16 nearby planetary nebulae are presented. Continued observations now give reduced errors and additional nebulae since initial results were given in 1997. Twelve nebulae have parallax errors less than 20 percent. Comparisons with other distance estimates are discussed.
\end{abstract}

Keywords. astrometry, stars: distances, planetary nebulae: general.

\section{Observations}

Measuring trigonometric parallaxes of the central stars of planetary nebulae (CSPN) can provide a check on other methods of determining distances for at least the nearest nebulae. Unfortunately, CSPN are near Hipparcos' faint magnitude limit, and the 19 observed had larger errors than typical for Hipparcos. With distances of several hundred pc for the nearest CSPN, parallax errors of $<1$ mas are essential for useful results.

Observing CSPN parallaxes at the Naval Observatory began in 1987, and initial results by Harris et al. 1997 were positive. The accuracy of parallaxes is continuing to improve for all types of stars, as shown in figure 1. Six additional CSPN have been added to the observing program since 1997, and the median error is now improved to 0.42 mas. A summary of the results is given in Table 1. A full description of this project and the results are given by Harris et al. 2006.

\section{Discussion}

Comparison with other distance determinations are shown in figure 2. Spectroscopic distances come mostly from Napiwotzki $(1999,2001)$. Spectroscopic distances are somewhat larger than parallax distances and must be overestimated for some stars, but they agree quite well after removing this systematic trend. The Hipparcos parallax for PHL932,

Table 1. Astrometric Results ${ }^{1}$

\begin{tabular}{lccclccc}
\hline PN & $\pi_{a b s}(\mathrm{mas})$ & $\mathrm{D}(\mathrm{pc})$ & $\mathrm{M}_{V}$ & $\mathrm{PN}$ & $\pi_{a b s}(\mathrm{mas})$ & $\mathrm{D}(\mathrm{pc})$ & $\mathrm{M}_{V}$ \\
\hline NGC 7293 & $4.56 \pm 0.49$ & 219 & 6.73 & Sh2-216 & $7.76 \pm 0.33$ & 129 & 6.83 \\
NGC 6853 & $2.64 \pm 0.33$ & 379 & 5.88 & PuWe1 & $2.74 \pm 0.31$ & 365 & 7.28 \\
NGC 6720 & $1.42 \pm 0.55$ & 704 & 6.26 & Ton320 & $1.88 \pm 0.33$ & 532 & 6.86 \\
A74 & $1.33 \pm 0.63$ & 752 & 7.29 & A21 & $1.85 \pm 0.51$ & 541 & 7.05 \\
RE1738+665 & $5.91 \pm 0.42$ & 169 & 8.29 & A7 & $1.48 \pm 0.42$ & 676 & 6.02 \\
DeHt5 & $3.34 \pm 0.56$ & 300 & 7.53 & A24 & $1.92 \pm 0.34$ & 521 & 8.61 \\
PHL932 & $3.36 \pm 0.62$ & 298 & 4.43 & A31 & $1.76 \pm 0.33$ & 568 & 6.53 \\
HDW4 & $4.78 \pm 0.40$ & 209 & 9.43 & PG1034+001 & $4.75 \pm 0.53$ & 211 & 6.43
\end{tabular}

${ }^{1}$ Complete results given by Harris et al. (2006).

${ }^{2}$ Includes average of USNO and HST parallax (Benedict et al. 2003). 

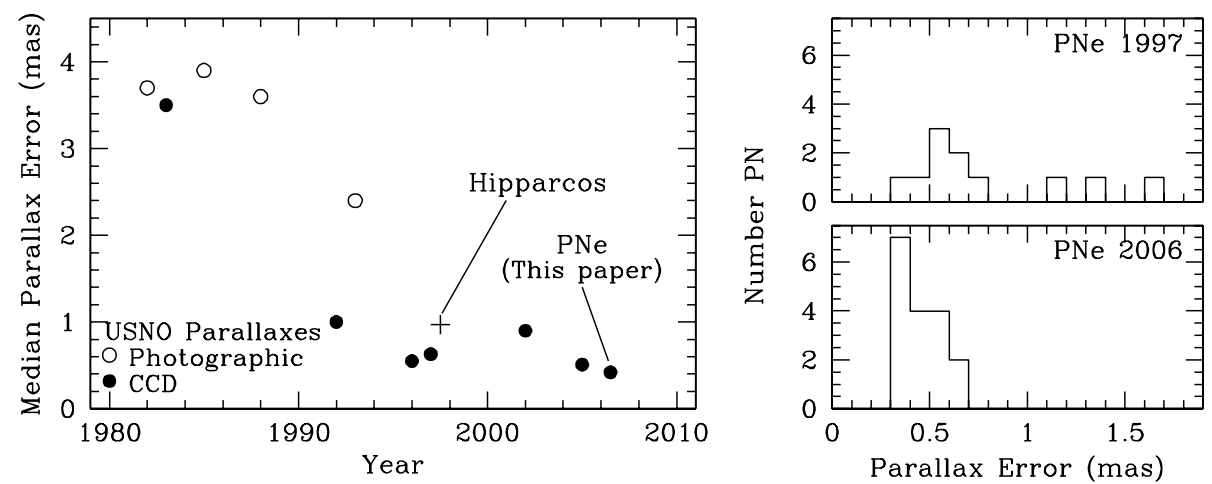

Figure 1. The historical improvement in accuracy of parallaxes from the Naval Observatory (left panel), and the improvement for PNe since 1997 (right panels).

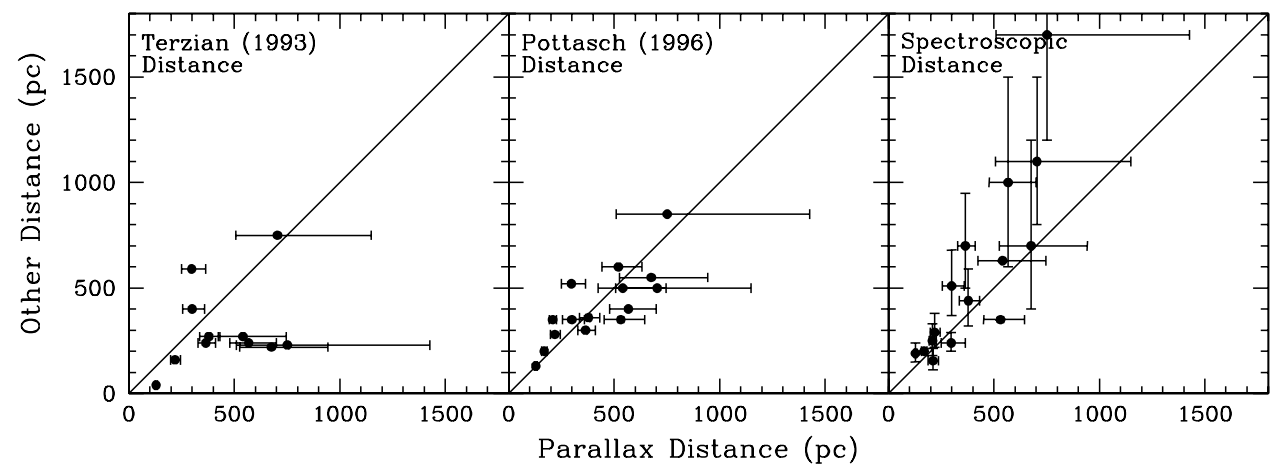

Figure 2. Comparison of parallax distances with three previous sources.

a peculiar object, is incorrect - it is an sdOB star, but may be in an unresolved binary with the true CSPN still not observed.

\section{Acknowledgements}

This USNO parallax work has been a group effort with participation by B. Canzian, C. Dahn, H. Guetter, S. Leggett, S. Levine, C. Luginbuhl, A. Monet, D. Monet, J. Pier, R. Stone, T. Tilleman, F. Vrba, and R. Walker.

\section{References}

Benedict, G.F., et al. 2003, AJ, 126, 2549

Harris, H.C., Dahn, C.C., Monet, D.G., \& Pier, J.R. 1997, in: H.J. Habing \& H.J.G.L.M Lamers (eds.), Planetary Nebulae, Proc. IAU Symposium No. 180 (Dordrecht: Kluwar), p. 40

Harris, H.C., et al. 2006, $A J$, in press

Napiwotzki, R. 1999, $A \mathscr{E} A$, 350, 101

Napiwotzki, R. 2001, $A \mathscr{E} A$, 367, 973

Pottasch, S.R. 1996, A\&A, 307, 561

Terzian, Y. 1993, in: R. Weinberger \& A. Acker (eds.), Planetary Nebulae, Proc. IAU Symposium No. 155 (Dordrecht: Kluwer), p. 109 